\title{
Towards the design principles of neural population codes
}

\author{
Elad Schneidman \\ Department of Neurobiology, Weizmann Institute of Science
}

\begin{abstract}
The ability to record the joint activity of large groups of neurons would allow for direct study of information representation and computation at the level of whole circuits in the brain. The combinatorial space of population activity patterns and neural noise imply that it would be impossible to directly map the relations between stimuli and population responses. Understanding of large neural population codes therefore depends on identifying simplifying design principles. We review recent results showing that strongly correlated population codes can be explained using minimal models that rely on low order relations among cells. We discuss the implications for large populations, and how such models allow for mapping the semantic organization of the neural codebook and stimulus space, and decoding.
\end{abstract}




\section{Redundancy reduction vs. robustness and learnability}

Efficient coding has been a key prospective design principle of the neural code - suggesting that neuronal spiking patterns convey information in a way that maximizes their capacity, given constraints such as energetic cost, number of neurons, time, etc. [1]. Accordingly, groups of neurons would maximize the information they convey about their stimuli by minimizing the overlap in the information they carry [2]. This idea of redundancy reduction implies that the responses of cells would be decorrelated, and was used for example, to predict or explain the nature of the receptive fields and response properties of cells in different parts of the visual system, the nature of eye movements, and more [3][7]

However, correlations among neurons are fundamental for robustness of the code to abundant neuronal noise. Moreover, redundancy is exactly what makes a code learnable from examples [8]. It is clear then that correlations must be an integral part of the design of the neural code. Indeed, analysis of large neuronal populations has shown that large groups can be strongly correlated (see below) and the potential importance and benefits have been extensively discussed [5], [9]-[13].

The design of the code of large populations of neurons in terms of correlation structures, connectivity, and sparseness have been explored theoretically (see e.g. [14]-[16]), but the ability to record simultaneously from large populations of cells makes it possible to study population codes directly [17]-[24]. Importantly, neuronal variability or noise means that in studying these codes we need to use probability distributions over the vast space of combinatorial coding patterns. Thus, we will not be able to exhaustively map the code of large populations by direct sampling, and would need to find simplifying principles that govern the organization of the codebook and its functional role [25]-[28].

\section{Correlation structures of population codes}

In many neural systems, the correlation between pairs of neurons is typically weak. This is the case for the correlation between cells averaged over stimuli (sometimes called signal correlations): denoting the spiking patterns of cells $i$ and $j$ with $x_{i}$ and $x_{j}, P\left(x_{i}, x_{j}\right)$ is typically close to $P\left(x_{i}\right) P\left(x_{j}\right)$ [11], [25], [29]. Similarly, the pairwise correlations in responses to repeated presentation of the same stimulus, s, are weak: $P\left(x_{i}, x_{j} \mid s\right)$ is typically not far from $P\left(x_{i} \mid s\right) P\left(x_{j} \mid s\right)$ [5], [30], [31], and so averaging over stimuli, cell pairs have been reported to have low "noise correlations" (A somewhat misleading name as the difference between these two distributions measures stimulus dependent coding correlations). Moreover, the information that pairs carry about their stimuli is typically weakly redundant: $I\left(x_{i}, x_{j} ; s\right)$ is typically lower than $I\left(x_{i} ; s\right)+I\left(x_{j} ; s\right)$ [30], [32], [33]. All these seem consistent with the idea of redundancy reduction. 
However, groups of a few tens of cells can be strongly correlated, even when their typical pairwise correlations are weak [25], [34]-[39]. These group correlations - quantified by the difference between $\mathrm{P}\left(x_{1}, x_{2}, \ldots, x_{n}\right)$ and $\mathrm{P}\left(x_{1}\right) \mathrm{P}\left(x_{2}\right) \ldots \mathrm{P}\left(x_{N}\right)$ - mean that the "vocabulary" of the neural population is more structured and less diverse than it would have been if the cells were independent, given the coding properties of single cells. Similarly, the stimulus dependent distribution of neural responses $\mathrm{P}\left(x_{1}, x_{2}, \ldots, x_{n} \mid s\right)$ can be far from the conditionally independent one $\mathrm{P}\left(x_{1} \mid s\right) \mathrm{P}\left(x_{2} \mid s\right) \ldots \mathrm{P}\left(x_{N} \mid s\right)$ for particular stimulus $s$, showing that while noise correlations may be weak on average, they can be strong for interesting stimuli and play a role in coding [40]-[42].

\section{Models of population activity reveal sparse high-order organization of the neural code}

To understand and quantify the nature and origin of the correlated structure of population codes, a few families of models have been commonly used. Generalized Linear Models (GLMs) extend the Linear-nonlinear Poisson rate models of single cells to predict the instantaneous rate of neuron $i$, given by $\lambda_{i}(t)=\exp \left(\boldsymbol{k}_{\boldsymbol{i}} \cdot \boldsymbol{s}+\boldsymbol{h}_{\boldsymbol{i}} \cdot \boldsymbol{x}_{\boldsymbol{i}}+\sum_{j} \boldsymbol{l}_{\boldsymbol{i} \boldsymbol{j}} \cdot \boldsymbol{x}_{\boldsymbol{j}}+\mu_{i}\right)$ where $\boldsymbol{s}$ is the stimulus, $\boldsymbol{x}_{\boldsymbol{j}}$ is the spiking history of cell $j$ at time $t$, and $\mu_{i}$ is the log of the cell's baseline firing rate, and $\boldsymbol{k}_{\boldsymbol{i}}$ is a spaio-temporal filter describing the cell's response to the stimulus, $\boldsymbol{l}_{\boldsymbol{i} \boldsymbol{j}}$, is the incoming coupling of cell $j$ to $i$, and $\boldsymbol{h}_{\boldsymbol{i}}$ is a temporal filter on the cell's own history; the last three are fit to maximize the likelihood of the model. These models surpass conditionally independent models in predicting the activity of single cells and low-order correlations between cells [43], [44], give a causal interpretation of the temporal patterns of cells, and have been successfully used in stimulus decoding [45]-[49]. The Maximum Entropy (ME) framework offers the mathematically minimal probabilistic model of population activity that satisfies a set of desired constraints without making arbitrary assumptions [50]. For example, if we denote the activity of cell $i$ in a small time bin by $x_{i}=0$ for silence, and $x_{i}=1$ for spiking, then the most random and unique model that obeys the firing rates and pairwise correlations between cells, is given by $P\left(x_{1}, x_{2}, \ldots, x_{n}\right)=$ $\frac{1}{Z} \exp \left\{\sum_{i} \alpha_{i} x_{i}+\frac{1}{2} \sum_{i j} \beta_{i j} x_{i} x_{j}\right\}$, where the set of $\alpha_{i}{ }^{\prime} s$ and $\beta_{i j}^{\prime} s \mathrm{w}$ are chosen to obey the constraints, and $Z$ is a normalization factor (or partition function). Such pairwise models capture with high accuracy the probability of individual spatial spiking patterns of tens of cells in different systems at the level of individual combinatorial patterns [25], [34], [35], [39], [51]-[54]. Restricted Boltzmann Machines offer a natural extension for capturing high-order relations between cells [55]. Figure 1 presents an example of the accuracy of pairwise maximum entropy models in capturing population activity patterns at the resolution of individual population patterns.

The accuracy of these models reflect the low-dimensional nature of population codes. For example, the accurate pairwise maximum entropy model relies on $n(n+1) / 2$ parameters, compared with the $2^{n}$ that would be needed for the 
distribution of binary activity patterns of $n$ cells in the general case, and the $n$ parameters needed for the independent model. Even these pairwise model may be further reduced while retaining accuracy by taking local structure or discretized interaction values into account [52].

For larger populations, an accurate model of the vocabulary of activity patterns may require models that go beyond pair, in particular for spatiotemporal patterns [36], [39], [53]-[56]. Adding a small number of high-order terms such as the distribution of synchrony values of the population or a selected set of high-order terms seem to be sufficient for $\sim 100$ cells responding to natural stimuli [53], [54], [57]. These high-order dependencies may arise from the nature of the stimulus, the internal structure of larger populations, or the activity of unseen (hidden) neurons [53], [55], [58], [59].

Extending this approach to the dynamics of the population and responses to stimuli, the stimulus-dependent maximum entropy models (SDME) of population encoding capture population responses significantly better than conditionally independent models (Fig. 2A). Moreover, these correlations become stronger with population size [40]-[42] (see e.g. Fig. 2B).

\section{Mapping the semantic space of population codes, and a neural metric on stimulus space}

The correlated activity of neuronal populations suggests that we should revise the classic view of the receptive field of a neuron with that of the collective response properties of populations [27], [28], [60]. To understand information representation and computation in large populations, we must go beyond detailed analysis of combinatorial coding patterns [61]-[63] to characterize the "semantic organization" of the spaces of stimuli and responses. Characterizing the noisy nature of mapping between stimuli and responses would enable deciphering the meaning of novel patterns that we have not seen before, and decode new stimuli that have been presented before. But how can we learn such neural dictionaries by generalizing from unavoidably severely undersampled set of observations of stimuli and responses, without making arbitrary assumptions?

Quantifying the similarity of population activity patterns $x_{i}$ and $x_{j}$ by their semantic overlap, namely, how similar are the stimuli these patterns are used to encode by comparing $p\left(s \mid x_{i}\right)$ and $p\left(s \mid x_{j}\right)$ - gives an assumption-free way to map the organization of the neural codebook (Fig. 3A). In the vertebrate retina this revealed that the neural codebook of groups of 20 cells is organized in a relatively small number of semantic clusters that capture almost all the information carried by the population (Fig. 3B-C). Importantly, metrics like Hamming distance, edit-distance, or other linear or bi-linear metrics fail to capture this organization of the space of population patterns [42]. 
On the decoding side, mean-squared-error (MSE) has been commonly used to measure how well sensory stimuli or movements are reconstructed from neural activity. Again, the similarity between two stimuli $s_{i}$ and $s_{j}$, can be quantified by the overlap of the neural responses they elicit, $p\left(x \mid s_{i}\right)$ and $p\left(x \mid s_{j}\right)$ - without the need of arbitrary assumptions of similarity. For the vertebrate retina this showed that similarity as judged by the retina was very different than what we would expect from Euclidean metrics, and MSE in particular [40], [41] (Fig. 2C).

Thus, the brain's "metrics" on stimulus space and on neural space are very different than our intuitive notion of similarity, and the clustered semantic organization of the space of neural activity patterns cannot be inferred from simple intuitive notions of what would constitute spiking similarities. Extending these ideas beyond sensory circuits and linking the brain metrics on stimuli and neural activity patterns would be necessary for our understanding of the code of high brain areas.

\section{Neural population codes of larger populations and cognitive processing}

Cognitive processing relies on computational architectures that are far more complicated than those of sensory circuits. Higher brain areas are usually built of many more cells, display a myriad of recurrent connections, and no apparent simple correlation structures. These imply intricate spatio-temporal processing which still empowers fast classification, decisions, and actions.

Experimental advances that would enable accurate recordings from thousands, millions, or all the neurons in an animal's brain at single spike resolution [18], [22], [64], would still suffer from the inability to quantify directly the full nature of correlations in large populations because of sampling issues. In many cases no specific population activity pattern would repeat itself over any experimental time window [53]. Can smaller networks reveal simplifying principles for large networks? The organization of the code of a few hundred cells in sensory systems already diverges from the view at the level of tens of cells - in terms of higher-order interactions [53], [54], [65], and the spatial decay of functional dependencies [52] — suggesting that there may be typical functional module size. A promising direction has been given by an example of learning the codebook of a large population by combining many models of small overlapping subnetworks, suggesting a hierarchical organization of learnable high-order codes [53], [66].

An especially intriguing feature of the vocabulary of neural populations is the distribution of frequencies of observed activity patterns, which resembles a power-law [53], [54]. While some of this behavior might be expected from the nature of collective responses with hidden elements (be it the stimulus or other neurons) [53], [59], [67], [68], it has been suggested to reflect design rather than epiphenomena of the code (see also [69]. Interestingly, this structure allows for learning the Reliable Interaction model (an approximate non-normalized maximum entropy model), which finds the dominant interactions of any order 
based on the frequent population patterns. In the retina, this model gave a very sparse high-order model with unparalleled accuracy in predicting the appearance of individual activity patterns in test data - showing that the code learnable from examples [53]. More broadly, the Zipf-like distribution of population states is reminiscent of physical systems near critical behavior, which is congruent with the possibility of collective behavior states of the code of large groups of neurons that allow for a wide range of distinct responses which would be easily decodable [65], [70]. Signatures of such critical behavior and its possible role as a feature of large networks in coding and adaptation have been presented in several different systems [65], [70], [71].

Merging these ideas of codebook generalization, sparse high-order interactions, semantic clusters, and the collective behavior of large populations, would be fundamental to our ability to understand the design principles of large networks and cognitive computations.

\section{What could the nature of spatiotemporal codes reveal about the cognitive computations they perform?}

The functional interactions between cells that underlie correlated codes also set a prior over population activity patterns, shaping the way stimuli would be perceived and encoded. Analysis of simple population models suggests that the interactions between cells that would maximize the information they carry about their stimuli, are the ones that would result in spontaneous activity that resembling the patterns that would be used to encode the stimulus [9], [72] — in accordance with experimental results of ongoing (spontaneous) cortical activity [37], [51], [73]-[76]. Moreover, rather than encoding specific stimuli, it has been suggested that neurons may be representing the likelihood of their inputs under an internally learned model [77]-[79]. Hierarchical population codes may allow for neurons to learn and encode these likelihoods as biological plausible models suggest [80]-[83].

Extending the models we presented here, for the spatio-temporal dynamics of population activity in high brain areas, would allow us to explore the computation or algorithms that these circuits implement. Ultimately, mapping the way population codes change or adapt on short time scales [84], [85] and during learning, would delineate the dynamics of individual cells and of groups in terms of stimulus features or information they learn to represent and compute [86], [87].

Finally, the ability to map the layout of connections in large networks [88] and to "put numbers" on these connections [89], [90], and a model based description of population activity patterns of the kind we presented here would hopefully allow for understanding the relations between neural architecture and function and the development of neural circuits, and would allow for tinkering and engineering of networks and neural computation. 


\section{Acknowledgements}

This research was supported by European Research Council grant \# 311238 NEURO-POPCODE, an Israel Science Foundation grant \#1629/12, Bi-national Science Foundation grant \# 2011058, and research support from Martin Kushner Schnur, Mexico and from Mr. and Mrs. Lawrence Feis, Winnetka, IL, USA. 
[1] H. B. Barlow, Sensory Communication, vol. 1. The MIT Press, 1962.

[2] J. J. Atick, "Could information theory provide an ecological theory of sensory processing?," Netw. Comput. Neural Syst., vol. 3, no. 2, pp. 213251, Jul. 1992.

[3] J. J. Atick and A. N. Redlich, "What Does the Retina Know about Natural Scenes?," Neural Comput., vol. 4, no. 2, pp. 196-210, Mar. 1992.

[4] I. Y. Segal, C. Giladi, M. Gedalin, M. Rucci, M. Ben-Tov, Y. Kushinsky, A. Mokeichev, and R. Segev, "Decorrelation of retinal response to natural scenes by fixational eye movements.," Proc. Natl. Acad. Sci. U. S. A., vol. 112, no. 10, pp. 3110-5, Mar. 2015.

[5] A. S. Ecker, P. Berens, G. a Keliris, M. Bethge, N. K. Logothetis, and A. S. Tolias, "Decorrelated neuronal firing in cortical microcircuits.," Science, vol. 327, no. 5965, pp. 584-587, 2010.

[6] W. E. Vinje and J. L. Gallant, "Sparse coding and decorrelation in primary visual cortex during natural vision.," Science, vol. 287, no. 5456, pp. 12736, Feb. 2000.

[7] Y. Dan, J. J. Atick, and R. C. Reid, "Efficient Coding of Natural Scenes in the Lateral Geniculate Nucleus: Experimental Test of a Computational Theory," J. Neurosci., vol. 16, no. 10, pp. 3351-3362, May 1996.

[8] H. Barlow, "Redundancy reduction revisited.," Network, vol. 12, no. 3, pp. 241-253, 2001.

[9] G. Tkacik, J. S. Prentice, V. Balasubramanian, and E. Schneidman, "Optimal population coding by noisy spiking neurons.," Proc. Natl. Acad. Sci. U. S. A., vol. 107, no. 32, pp. 14419-14424, 2010.

[10] B. B. Averbeck, P. E. Latham, and A. Pouget, "Neural correlations, population coding and computation.," Nat. Rev. Neurosci., vol. 7, no. 5, pp. 358-366, 2006.

[11] M. R. Cohen and A. Kohn, "Measuring and interpreting neuronal correlations.," Nat. Neurosci., vol. 14, no. 7, pp. 811-819, 2011.

[12] S. Panzeri, G. Pola, F. Petroni, M. P. Young, and R. S. Petersen, "A critical assessment of different measures of the information carried by correlated neuronal firing.," Biosystems., vol. 67, no. 1-3, pp. 177-85, 2002.

[13] E. Schneidman, W. Bialek, and M. J. Berry, "Synergy, redundancy, and independence in population codes.," J. Neurosci., vol. 23, no. 37, pp. 11539-53, Dec. 2003.

[14] L. F. Abbott and P. Dayan, "The effect of correlated variability on the accuracy of a population code.," Neural Comput., vol. 11, no. 1, pp. 91101, 1999.

[15] B. a Olshausen and D. J. Field, "Emergence of simple-cell receptive field properties by learning a sparse code for natural images.," Nature, vol. 381, no. 6583. pp. 607-609, 1996.

[16] K. Kang and H. Sompolinsky, "Mutual information of population codes and distance measures in probability space," Phys. Rev. Lett., vol. 86, no. 21, pp. 4958-4961, 2001.

[17] M. B. Ahrens, M. B. Orger, D. N. Robson, J. M. Li, and P. J. Keller, "Whole- 
brain functional imaging at cellular resolution using light-sheet microscopy," Nat Methods, vol. 10, no. 5, pp. 413-420, 2013.

[18] R. Prevedel, Y.-G. Yoon, M. Hoffmann, N. Pak, G. Wetzstein, S. Kato, T. Schrödel, R. Raskar, M. Zimmer, E. S. Boyden, and A. Vaziri, "Simultaneous whole-animal 3D imaging of neuronal activity using lightfield microscopy.," Nat. Methods, vol. 11, no. 7, pp. 727-30, 2014.

[19] T. A. Szuts, V. Fadeyev, S. Kachiguine, A. Sher, M. V Grivich, M. Agrochão, P. Hottowy, W. Dabrowski, E. V Lubenov, A. G. Siapas, N. Uchida, A. M. Litke, and M. Meister, "A wireless multi-channel neural amplifier for freely moving animals.," Nat. Neurosci., vol. 14, no. 2, pp. 263-9, Feb. 2011.

[20] D. Aronov and D. W. Tank, "Engagement of neural circuits underlying 2D spatial navigation in a rodent virtual reality system," Neuron, vol. 84, no. 2, pp. 442-456, 2014.

[21] A. Berényi, Z. Somogyvári, A. J. Nagy, L. Roux, J. D. Long, S. Fujisawa, E. Stark, A. Leonardo, T. D. Harris, and G. Buzsáki, "Large-scale, highdensity (up to 512 channels) recording of local circuits in behaving animals.," J. Neurophysiol., vol. 111, no. 5, pp. 1132-49, Mar. 2014.

[22] D. R. Hochbaum, Y. Zhao, S. L. Farhi, N. Klapoetke, C. a Werley, V. Kapoor, P. Zou, J. M. Kralj, D. Maclaurin, N. Smedemark-Margulies, J. L. Saulnier, G. L. Boulting, C. Straub, Y. K. Cho, M. Melkonian, G. K.-S. Wong, D. J. Harrison, V. N. Murthy, B. L. Sabatini, E. S. Boyden, R. E. Campbell, and A. E. Cohen, "All-optical electrophysiology in mammalian neurons using engineered microbial rhodopsins.," Nat. Methods, no. May, pp. 1-34, 2014.

[23] Y. Ziv, L. D. Burns, E. D. Cocker, E. O. Hamel, K. K. Ghosh, L. J. Kitch, A. El Gamal, and M. J. Schnitzer, "Long-term dynamics of CA1 hippocampal place codes.," Nat. Neurosci., vol. 16, no. 3, pp. 264-6, Mar. 2013.

[24] J. Freeman, G. D. Field, P. H. Li, M. Greschner, D. E. Gunning, K. Mathieson, A. Sher, A. M. Litke, L. Paninski, E. P. Simoncelli, and E. Chichilnisky, "Mapping nonlinear receptive field structure in primate retina at single cone resolution," Elife, vol. 4, p. e05241, Oct. 2015.

[25] E. Schneidman, M. J. Berry, R. Segev, and W. Bialek, "Weak pairwise correlations imply strongly correlated network states in a neural population.," Nature, vol. 440, no. 7087, pp. 1007-12, 2006.

[26] J. P. Cunningham and B. M. Yu, "Dimensionality reduction for large-scale neural recordings.," Nat. Neurosci., vol. 17, no. 11, pp. 1500-9, Nov. 2014. ${ }^{*}$ ) Reviews dimensionality reduction approaches to characterization of large neural population activity patterns

[27] W. Singer, "Cortical dynamics revisited.," Trends Cogn. Sci., vol. 17, no. 12, pp. 616-26, Dec. 2013.

[28] G. Buzsáki, "Neural syntax: cell assemblies, synapsembles, and readers.," Neuron, vol. 68, no. 3, pp. 362-85, Nov. 2010.

[29] M. A. Smith and A. Kohn, "Spatial and temporal scales of neuronal correlation in primary visual cortex.," J. Neurosci., vol. 28, no. 48, pp. 12591-603, Nov. 2008. 
[30] J. L. Puchalla, E. Schneidman, R. a. Harris, and M. J. Berry, "Redundancy in the population code of the retina," Neuron, vol. 46, pp. 493-504, 2005.

[31] J. Cafaro and F. Rieke, "Noise correlations improve response fidelity and stimulus encoding.," Nature, vol. 468, no. 7326, pp. 964-7, Dec. 2010.

[32] G. Pola, a Thiele, K. P. Hoffmann, and S. Panzeri, "An exact method to quantify the information transmitted by different mechanisms of correlational coding.," Network, vol. 14, no. 1, pp. 35-60, 2003.

[33] D. S. Reich, F. Mechler, and J. D. Victor, "Independent and redundant information in nearby cortical neurons.," Science, vol. 294, no. 5551, pp. 2566-2568, 2001.

[34] J. Shlens, G. D. Field, J. L. Gauthier, M. I. Grivich, D. Petrusca, A. Sher, A. M. Litke, and E. J. Chichilnisky, "The structure of multi-neuron firing patterns in primate retina.," J. Neurosci., vol. 26, no. 32, pp. 8254-8266, 2006.

[35] J. Shlens, G. D. Field, J. L. Gauthier, M. Greschner, A. Sher, A. M. Litke, and $\mathrm{E}$. J. Chichilnisky, "The structure of large-scale synchronized firing in primate retina.," J. Neurosci., vol. 29, no. 15, pp. 5022-5031, 2009.

[36] O. Marre, S. El Boustani, Y. Frégnac, and A. Destexhe, "Prediction of Spatiotemporal Patterns of Neural Activity from Pairwise Correlations," Phys. Rev. Lett., vol. 102, no. 13, p. 138101, 2009.

[37] P. Berkes, G. Orbán, M. Lengyel, and J. Fiser, "Spontaneous cortical activity reveals hallmarks of an optimal internal model of the environment.," Science (80-. )., vol. 331, no. 6013, pp. 83-87, 2011.

$\left({ }^{*}\right)$ Show that with age spontaneous and stimulus driven cortical population activity become more similar, suggesting spontaneous activity serving as a prior over neural responses

[38] W. Truccolo, L. R. Hochberg, and J. P. Donoghue, "Collective dynamics in human and monkey sensorimotor cortex: predicting single neuron spikes.," Nat. Neurosci., vol. 13, no. 1, pp. 105-111, 2010.

(*) Show that spiking history of small and randomly sampled ensembles of cortical neurons could predict subsequent single neuron spiking with high accuracy and better than by the ensemble's instantaneous state

[39] A. Tang, D. Jackson, J. Hobbs, W. Chen, J. L. Smith, H. Patel, A. Prieto, D. Petrusca, M. I. Grivich, A. Sher, P. Hottowy, W. Dabrowski, A. M. Litke, and J. M. Beggs, "A maximum entropy model applied to spatial and temporal correlations from cortical networks in vitro.," J. Neurosci., vol. 28, no. 2, pp. 505-518, 2008.

[40] E. Granot-Atedgi, G. Tkačik, R. Segev, and E. Schneidman, "Stimulusdependent Maximum Entropy Models of Neural Population Codes," PLOS Comput Biol, vol. 9, no. 3, p. e1002922, 2013.

[41] G. Tkačik, E. Granot-Atedgi, R. Segev, and E. Schneidman, "Retinal metric: A stimulus distance measure derived from population neural responses," Phys. Rev. Lett., vol. 110, no. 5, pp. 1-5, 2013.

(*)Present a way to learn a neural based metric on stimulus space, and show it is significantly different from commonly used Euclidean metrics

[42] E. Ganmor, R. Segev, and E. Schneidman, "A thesaurus for a neural 
population code," Elife, vol. 4, p. e06134, Sep. 2015.

$\left({ }^{*}\right)$ Show that that population codebook is organized as a set of semantic clusters, which cannot be inferred from syntactic metrics on population patterns, and that most of the information carried by neuronal population is captured by the cluster a pattern belongs to.

[43] J. W. Pillow, J. Shlens, L. Paninski, A. Sher, A. M. Litke, E. J. Chichilnisky, and E. P. Simoncelli, "Spatio-temporal correlations and visual signalling in a complete neuronal population.," Nature, vol. 454, no. 7207, pp. 995-999, 2008.

[44] W. Truccolo, U. T. Eden, M. R. Fellows, J. P. Donoghue, and E. N. Brown, "A point process framework for relating neural spiking activity to spiking history, neural ensemble, and extrinsic covariate effects.," J. Neurophysiol., vol. 93, no. 2, pp. 1074-89, Feb. 2005.

[45] J. W. Pillow, Y. Ahmadian, and L. Paninski, "Model-based decoding, information estimation, and change-point detection techniques for multineuron spike trains.," Neural Comput., vol. 23, no. 1, pp. 1-45, 2011.

$\left(^{\star}\right)$ Present efficiently learnable families of models that predict population responses to stimuli and used for stimulus decoding

[46] J. W. Pillow, L. Paninski, V. J. Uzzell, E. P. Simoncelli, and E. J. Chichilnisky, "Prediction and decoding of retinal ganglion cell responses with a probabilistic spiking model.," J. Neurosci., vol. 25, no. 47, pp. 11003-11013, 2005.

[47] Y. Ahmadian, J. W. Pillow, and L. Paninski, "Efficient Markov chain Monte Carlo methods for decoding neural spike trains.," Neural Comput., vol. 23, no. 1, pp. 46-96, 2011.

[48] E. N. Brown, L. M. Frank, D. Tang, M. C. Quirk, and M. A. Wilson, "A Statistical Paradigm for Neural Spike Train Decoding Applied to Position Prediction from Ensemble Firing Patterns of Rat Hippocampal Place Cells," J. Neurosci., vol. 18, no. 18, pp. 7411-7425, Sep. 1998.

[49] E. N. Brown, R. E. Kass, and P. P. Mitra, "Multiple neural spike train data analysis: state-of-the-art and future challenges.," Nat. Neurosci., vol. 7, no. 5, pp. 456-61, 2004.

[50] E. T. Jaynes, "Information Theory and Statistical Mechanics," Phys. Rev., vol. 106, no. 4, pp. 620-630, May 1957.

[51] O. Marre, P. Yger, A. P. Davison, and Y. Frégnac, "Reliable recall of spontaneous activity patterns in cortical networks.," J. Neurosci., vol. 29, no. 46, pp. 14596-14606, 2009.

[52] E. Ganmor, R. Segev, and E. Schneidman, "The architecture of functional interaction networks in the retina.," J. Neurosci., vol. 31, no. 8, pp. 30443054, 2011.

[53] E. Ganmor, R. Segev, and E. Schneidman, "Sparse low-order interaction network underlies a highly correlated and learnable neural population code.," Proc. Natl. Acad. Sci. U. S. A., vol. 108, no. 23, pp. 9679-84, 2011. $\left.{ }^{*}\right)$ Show that high-order interactions are needed to describe the vocabulary of large neural populations, and a way to learn a highly sparse set of these interactions, suggesting the code is learnable 
[54] G. Tkačik, O. Marre, D. Amodei, E. Schneidman, W. Bialek, and M. J. Berry, "Searching for collective behavior in a large network of sensory neurons.," PLoS Comput. Biol., vol. 10, no. 1, p. e1003408, Jan. 2014.

[55] U. Köster, J. Sohl-Dickstein, C. M. Gray, and B. A. Olshausen, "Modeling higher-order correlations within cortical microcolumns.," PLoS Comput. Biol., vol. 10, no. 7, p. e1003684, Jul. 2014.

$\left.{ }^{*}\right)$ Compare different families of models to capture the high order structure of cortical population activity patterns

[56] S.-I. Amari and H. Nakahara, "Correlation and independence in the neural code," Neural Comput., vol. 18, no. 6, pp. 1259-1267, 2006.

[57] G. Tkacik, O. Marre, T. Mora, D. Amodei, M. J. Berry, and W. Bialek, "The simplest maximum entropy model for collective behavior in a neural network," J. Stat. ..., no. 1, pp. 1-5, 2012.

[58] J. H. Macke, M. Opper, and M. Bethge, "Common Input Explains HigherOrder Correlations and Entropy in a Simple Model of Neural Population Activity," Phys. Rev. Lett., vol. 106, no. 20, p. 208102, 2011.

[59] E. Schneidman, S. Still, M. J. Berry, and W. Bialek, "Network information and connected correlations.," Phys. Rev. Lett., vol. 91, no. 23, p. 238701, 2003.

[60] R. Yuste, "From the neuron doctrine to neural networks," Nat. Rev. Neurosci., vol. 16, no. 8, pp. 487-497, 2015.

$\left.{ }^{*}\right)$ Reviews the need to change our view of neuronal function and encoding from single neurons to ensembles

[61] L. C. Osborne, S. E. Palmer, S. G. Lisberger, and W. Bialek, "The neural basis for combinatorial coding in a cortical population response.," $J$.

Neurosci., vol. 28, no. 50, pp. 13522-13531, 2008.

[62] E. Schneidman, J. L. Puchalla, R. Segev, R. A. Harris, W. Bialek, and M. J. Berry II, "Synergy from Silence in a Combinatorial Neural Code," J. Neurosci., vol. 31, no. 44, pp. 15732-15741, 2011.

[63] N. Brenner, S. P. Strong, R. Köberle, W. Bialek, and R. R. de R. van Steveninck, "Synergy in a Neural Code," Neural Comput., vol. 12, no. 7, pp. 1531-1552, 2000.

[64] P. J. Keller and M. B. Ahrens, "Visualizing Whole-Brain Activity and Development at the Single-Cell Level Using Light-Sheet Microscopy," Neuron, vol. 85, no. 3, pp. 462-483, 2015.

[65] G. Tkačik, T. Mora, O. Marre, D. Amodei, S. E. Palmer, M. J. Berry, and W. Bialek, "Thermodynamics and signatures of criticality in a network of neurons.," Proc. Natl. Acad. Sci. U. S. A., vol. 112, no. 37, pp. 11508-13, Sep. 2015.

$\left.{ }^{*}\right)$ Present the divergence of codebook properties for large neural populations, suggesting critical behavior of the code

[66] D. Soudry, S. Keshri, P. Stinson, M.-H. Oh, G. Iyengar, and L. Paninski, "Efficient 'Shotgun' Inference of Neural Connectivity from Highly Subsampled Activity Data.," PLoS Comput. Biol., vol. 11, no. 10, p. e1004464, Oct. 2015.

[67] D. J. Schwab, I. Nemenman, and P. Mehta, "Zipf's law and criticality in 
multivariate data without fine-tuning," Phys. Rev. Lett., vol. 113, no. 6, pp. 1-5, 2014.

[68] L. Aitchison, N. Corradi, and P. E. Latham, "Zipf's law arises naturally in structured, high-dimensional data," Jul. 2014.

[69] G. Buzsáki and K. Mizuseki, "The log-dynamic brain: how skewed distributions affect network operations.," Nat. Rev. Neurosci., vol. 15, no. 4, pp. 264-78, Apr. 2014.

[70] C. Tetzlaff, S. Okujeni, U. Egert, F. Wörgötter, and M. Butz, "SelfOrganized Criticality in Developing Neuronal Networks," PLoS Comput Biol, vol. 6, no. 12, p. e1001013, 2010.

[71] T. Mora, S. Deny, and O. Marre, "Dynamical criticality in the collective activity of a population of retinal neurons," Phys. Rev. Lett., vol. 114, no. 7, p. 078105, 2015.

[72] P. Berkes, G. Orbán, M. Lengyel, and J. Fiser, "Spontaneous cortical activity reveals hallmarks of an optimal internal model of the environment.," Science, vol. 331, no. 6013, pp. 83-7, Jan. 2011.

[73] A. Arieli, A. Sterkin, A. Grinvald, and A. Aertsen, "Dynamics of ongoing activity," Science (80-. )., vol. 273, pp. 1868-1871, 1996.

[74] M. Tsodyks, T. Kenet, a Grinvald, and a Arieli, "Linking spontaneous activity of single cortical neurons and the underlying functional architecture.," Science, vol. 286, no. 5446, pp. 1943-1946, 1999.

[75] A. Luczak, P. Barthó, and K. D. Harris, "Spontaneous events outline the realm of possible sensory responses in neocortical populations," Neuron, vol. 62, no. 3, pp. 413-425, 2009.

[76] J. -e. K. Miller, I. Ayzenshtat, L. Carrillo-Reid, and R. Yuste, "Visual stimuli recruit intrinsically generated cortical ensembles," Proc. Natl. Acad. Sci., vol. 111, no. 38, pp. E4053-E4061, Sep. 2014.

$\left.{ }^{*}\right)$ Present the relations between stimulus-induced ensemble responses and spontaneous population activity, and the dynamic nature of ensemble membership in stimulus encoding

[77] R. S. Zemel, P. Dayan, and A. Pouget, "Probabilistic interpretation of population codes.," Neural Comput., vol. 10, no. 2, pp. 403-30, Feb. 1998.

[78] S. Deneve, P. E. Latham, and a Pouget, "Efficient computation and cue integration with noisy population codes.," Nat. Neurosci., vol. 4, no. 8, pp. 826-831, 2001.

[79] T. Yang and M. N. Shadlen, "Probabilistic reasoning by neurons.," Nature, vol. 447, no. 7148, pp. 1075-80, Jun. 2007.

[80] S. Deneve, "Bayesian spiking neurons I: inference.," Neural Comput., vol. 20, no. 1, pp. 91-117, Jan. 2008.

[81] S. Deneve, "Bayesian spiking neurons II: learning.," Neural Comput., vol. 20, no. 1, pp. 118-45, Jan. 2008.

[82] P. O. Hoyer and A. Hyvärinen, "Interpreting Neural Response Variability as Monte Carlo Sampling of the Posterior," in Advances in Neural Information Processing Systems, 2003, pp. 293-300.

[83] L. Buesing, J. Bill, B. Nessler, and W. Maass, "Neural dynamics as sampling: a model for stochastic computation in recurrent networks of 
spiking neurons.," PLoS Comput. Biol., vol. 7, no. 11, p. e1002211, Nov. 2011.

[84] D. A. Gutnisky and V. Dragoi, "Adaptive coding of visual information in neural populations.," Nature, vol. 452, no. 7184, pp. 220-4, Mar. 2008.

[85] S. E. Palmer, O. Marre, M. J. Berry, and W. Bialek, "Predictive information in a sensory population," Proc. Natl. Acad. Sci., vol. 112, no. 22, pp. 69086913, Jun. 2015.

$\left.{ }^{*}\right)$ Analyze the nature of predictive coding by population of cells based on stimulus statistics

[86] N. D. Goodman, J. B. Tenenbaum, J. Feldman, and T. L. Griffiths, "A rational analysis of rule-based concept learning.," Cogn. Sci., vol. 32, no. 1, pp. 108-154, 2008.

[87] Y. Cohen and E. Schneidman, "High-order feature-based mixture models of classification learning predict individual learning curves and enable personalized teaching," Proc. Natl. Acad. Sci., vol. 110, no. 2, pp. 684-689, 2013.

[88] M. Helmstaedter, K. L. Briggman, S. C. Turaga, V. Jain, H. S. Seung, and W. Denk, "Connectomic reconstruction of the inner plexiform layer in the mouse retina," Nature, vol. 500, no. 7461, pp. 168-174, Aug. 2013.

[89] L. Cossell, M. F. lacaruso, D. R. Muir, R. Houlton, E. N. Sader, H. Ko, S. B. Hofer, and T. D. Mrsic-Flogel, "Functional organization of excitatory synaptic strength in primary visual cortex," Nature, vol. 518, no. 7539, pp. 399-403, Feb. 2015.

[90] X. Jiang, S. Shen, C. R. Cadwell, P. Berens, F. Sinz, A. S. Ecker, S. Patel, and A. S. Tolias, "Principles of connectivity among morphologically defined cell types in adult neocortex," Science (80-. )., vol. 350, no. 6264, pp. aac9462-aac9462, Nov. 2015.

${ }^{*}$ )Present large-scale, comprehensive profiling of neocortical neurons and their type-dependent characteristic input-output connectivity profile 
Fig. 1. Example of the high accuracy of a pairwise maximum entropy models for spatial activity patterns of a large network responding to natural stimuli, and signs of higher order interactions. A. A segment of the simultaneous action potential sequences of 99 ganglion cells in the salamander retina responding to a natural movie clip. Each line corresponds to a single neuron, each tick represents a single spike. B. The probability of occurrence of each simultaneous (spatial) population activity pattern that appeared in the experiment as predicted if all cells were independent $\left(P^{(1)}\right.$, grey), or by the 2 nd order maximum entropy model, which takes into account pairwise correlations ( $P$ (2) , red), are plotted against the measured rate. Note that while most rare patterns fall within the confidence region (not shown), frequently observed patterns are misestimated by the pairwise model. Adding a small number of highorder dependencies in this case gives a nearly perfect fit (not shown). C. Probability distribution of synchronous spiking events in the 99 cell population in response to a long natural movie (black). The distribution of synchronous events for the same 99 cells predicted by the independent model (grey, $\left.P^{(1)}\right)$, and the synchrony distribution predicted from the 2 nd order maximum entropy model $\mathrm{P}(2)$ (red) are shown D. Probability distribution of synchronous spiking events in the 99 cell population in response to artificial white noise stimuli (black). 2nd order model, and independent model, built as described in $\mathrm{C}$. Unlike the responses to natural movies, the pairwise model provides a very good fit to responses to white noise stimuli, reflecting a negligible role for higher order interactions in the population activity evoked by such stimuli. (Adapted from [53]).

Fig. 2 Stimulus dependent maximum entropy model captures noise correlations between cells, and allow for learning a metric on stimulus space, based on the neural population. A. Pairwise SDME model predicts population activity patterns for $\mathrm{N}=100$ retinal ganglion cells responding to Gaussian white noise stimulus much better than conditionally independent model. The log-likelihood ratio of the population firing patterns under the SDME model and under the conditional independent model, shown as a function of time (red dots, scale at left) for an example stimulus repeat (models fitted on train data; example shown is for test data). For reference, the average time dependent population firing rate is shown in grey (scale at right). B. The performance of the SDME model relative to conditionally independent LN models. The average log likelihood ratio between the SDME and the conditional independent models evaluated on the test set, as a function of the population size, $\mathrm{N}$ (error bars = std over 10 randomly chosen groups of neurons at that $\mathrm{N}$ ). C. An example of learning a metric on stimulus space from population neural responses: Stimulus segment with two $400 \mathrm{~ms}$ stimulus clips s 1 (red), s2 (blue). Bottom: For every pair of time bins in the experiment, the Euclidean distance $D_{2}$ between the corresponding stimulus clips is shown in the upper diagonal part of the matrix, and the retinal neurons' distance $D_{\text {ret }}$ in the lower part. (Adapted from [40], [41]). 
Fig. 3. Mapping the semantic organization of population codebook, reveals a small set of semantic clusters. A. A cartoon of the way the semantic similarity between neural activity patterns was estimated. For two neural words, we inferred the distribution over stimuli given the neural word, using an SDME model for the neural population response. Then the similarity between patterns was estimated using the Jensen-Shannon divergence between the distributions, d. B. The neural codebook of the retina is comprised of distinct clustered responses with highly similar meaning. Example of the similarity matrix of the population responses of representative group of 20 neurons to a natural movie. Each entry in the matrix corresponds to the similarity d between two population responses ("words") observed in the test data. Matrix rows (and columns) are ordered according to the result of clustering the words using k-means. On the bottom we show the population responses corresponding to the entries in the matrix; the blue lines mark borders between different clusters. C. A graphical representation of the organization of the codebook of these cells: The responses belonging to clusters that contain 30-300 patterns were embedded in 3D using a dimensionality reduction algorithm (The Euclidean distance in the plot approximates the similarity measure $d$ ). Each ellipse represents the 1 STD Gaussian fit to all responses belonging to a single cluster. The coordinates also correspond to the RGB value of each ellipse, thus nearby clusters share similar colors. (Adapted from [42]). 


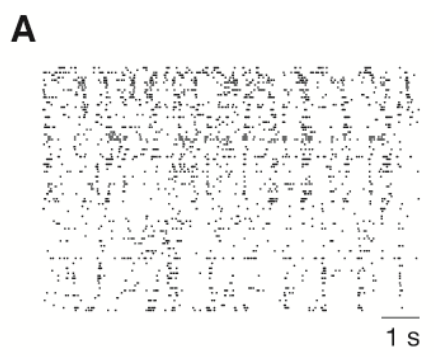

B

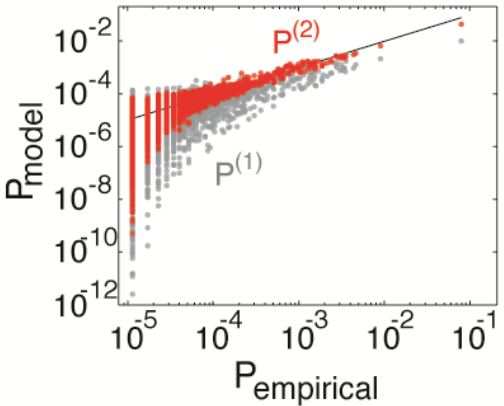

C

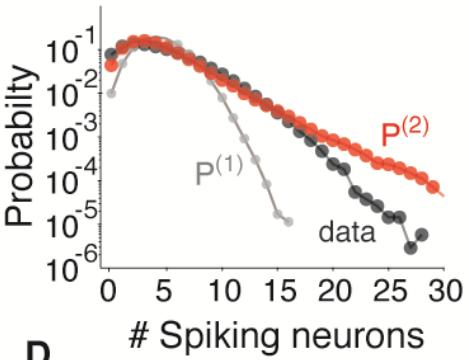

D

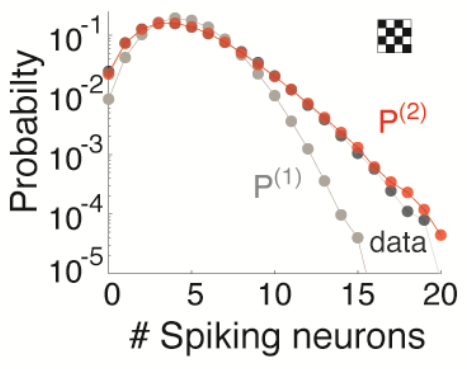



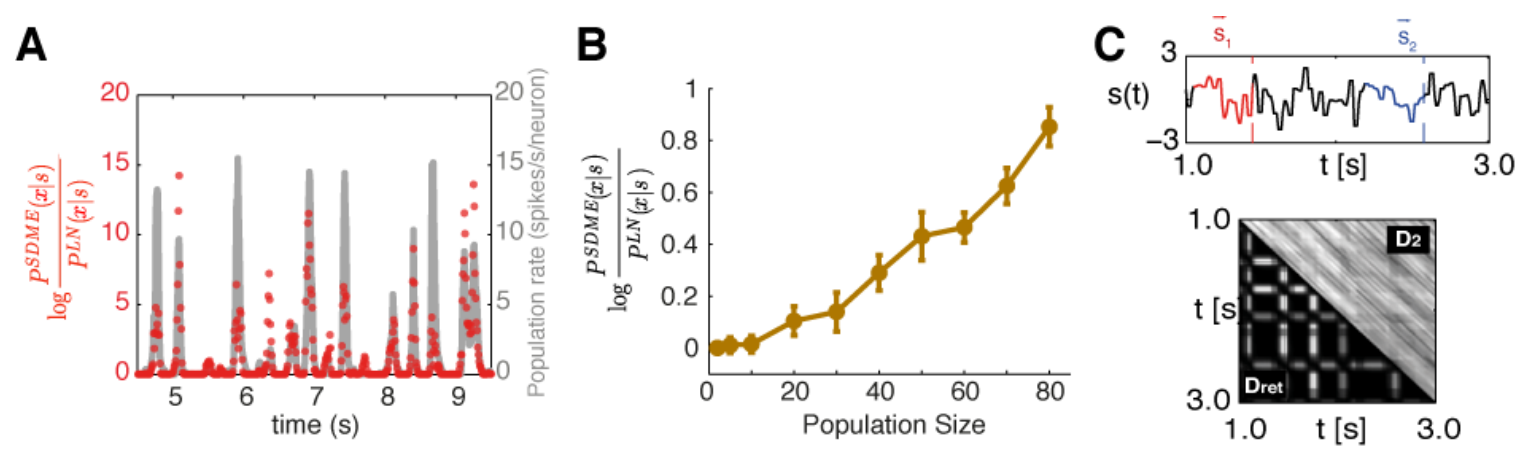
A
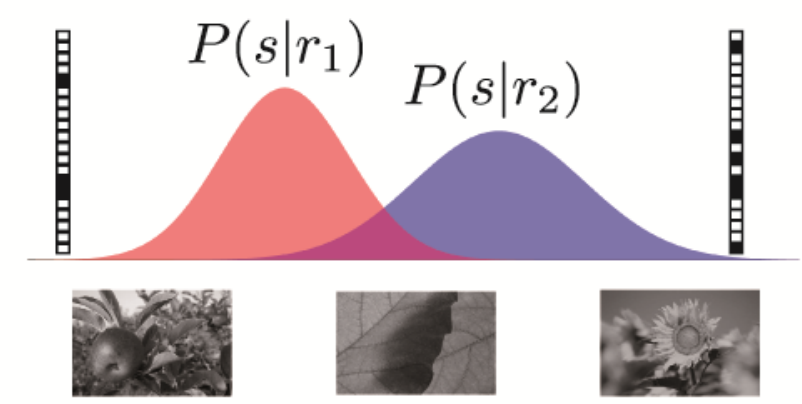

$$
d\left(r_{1}, r_{2}\right)=D_{J S}\left(P\left(s \mid r_{1}\right) \| P\left(s \mid r_{2}\right)\right)
$$

B

$$
\mathrm{D}_{\text {JS }} \text { (bits) }
$$
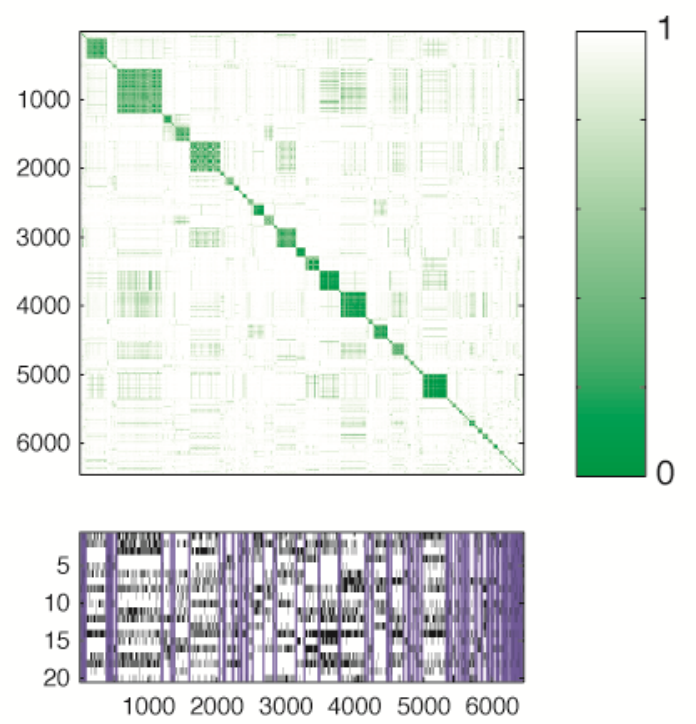

B

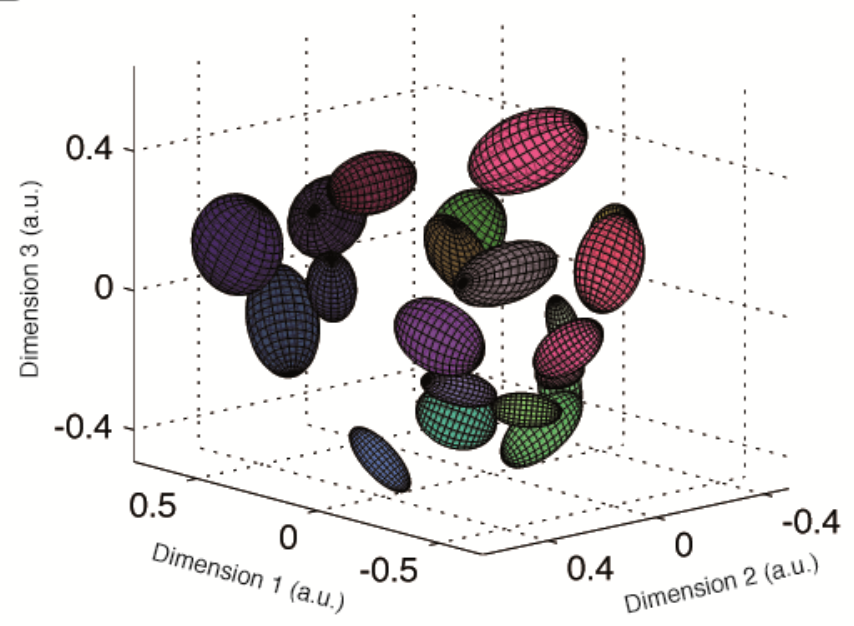

